\title{
EW and radiative penguin transitions from B-factories
}

\author{
Simon Akar*i \\ CPPM/CNRS/Aix-Marseille Université \\ E-mail: akar@cppm.in2p3.fr
}

\begin{abstract}
We present recent results from the $B A B A R$ and Belle Collaborations on electroweak and radiative decays. These include searches for new physics via measurements of several observables in the angular analyses of $B \rightarrow K^{*} \ell^{+} \ell^{-}$decays, as well as searches for the rare $B^{0} \rightarrow \phi \gamma$ and $B^{+} \rightarrow$ $K^{+} \tau^{+} \tau^{-}$decays.
\end{abstract}

16th International Conference on B-Physics at Frontier Machines

2-6 May 2016

Marseille, France

*Speaker.

${ }^{\dagger}$ On behalf of the BABAR and Belle Collaborations. 


\section{Introduction}

The BABAR and Belle experiments recorded $e^{+} e^{-}$collisions at the $\Upsilon(4 S)$ resonance with integrated luminosities of $429 \mathrm{fb}^{-1}$ and $711 \mathrm{fb}^{-1}$, respectively, corresponding to $471 \times 10^{6}$ and $772 \times 10^{6} B \bar{B}$ pairs produced. In the LHC era, the $B$ factories are still competitive, especially for channels involving neutral particles such as $\pi^{0}$ or $K_{S}^{0}$, and $\tau$ leptons. We present here a selection of recent results on electroweak and radiative decays from the BABAR and Belle experiments. In the Standard Model (SM), both the $b \rightarrow s$ and $b \rightarrow d$ transitions are quark-level flavor-changing neutral current (FCNC) processes. Since all FCNC processes are forbidden at tree level in the SM, the lowest order diagrams representing these transitions must involve loops. In such processes, QCD corrections can typically be described using an effective Hamiltonian defined as $\mathrm{H}_{\mathrm{eff}} \propto \sum_{i=1}^{10} C_{i} O_{i}$, where the $C_{i}$ and $O_{i}$ are, respectively, the short-distance Wilson coefficients (WC) and local longdistance operators. Contributions from New Physics (NP) in $b \rightarrow s(d)$ transitions may modify the SM values of the WC, and change the values, predicted by the SM, of observables such as branching fractions and $C P$ asymmetries as well as angular distributions.

\section{Angular analyses of $B \rightarrow K^{*} \ell^{+} \ell^{-}$decays}

The analysis of the rare decay $B \rightarrow K^{*} \ell^{+} \ell^{-}$, where $\ell^{+} \ell^{-}$is hereafter $e^{+} e^{-}$or $\mu^{+} \mu^{-}$, gives access to several angular observables that are sensitive to NP contributions. The decay is fully defined by three helicity angles $\vec{\Omega}=\left(\theta_{l}, \theta_{K}, \phi\right)$, and $q^{2}$, the invariant mass of the dilepton system squared. The $C P$-averaged angular distribution in a bin of $q^{2}$ is given by [1]

$$
\begin{aligned}
\frac{1}{\mathrm{~d}(\Gamma+\bar{\Gamma}) / \mathrm{d} q^{2}} \frac{\mathrm{d}^{3}(\Gamma+\bar{\Gamma})}{\mathrm{d} \vec{\Omega}}=\frac{9}{32 \pi} & {\left[\frac{3}{4}\left(1-F_{\mathrm{L}}\right) \sin ^{2} \theta_{K}+F_{\mathrm{L}} \cos ^{2} \theta_{K}+\frac{1}{4}\left(1-F_{\mathrm{L}}\right) \sin ^{2} \theta_{K} \cos 2 \theta_{l}\right.} \\
& -F_{\mathrm{L}} \cos ^{2} \theta_{K} \cos 2 \theta_{l}+S_{3} \sin ^{2} \theta_{K} \sin ^{2} \theta_{l} \cos 2 \phi \\
& +S_{4} \sin 2 \theta_{K} \sin 2 \theta_{l} \cos \phi+S_{5} \sin 2 \theta_{K} \sin \theta_{l} \cos \phi \\
& +\frac{4}{3} A_{\mathrm{FB}} \sin ^{2} \theta_{K} \cos \theta_{l}+S_{7} \sin 2 \theta_{K} \sin \theta_{l} \sin \phi \\
& \left.+S_{8} \sin 2 \theta_{K} \sin 2 \theta_{l} \sin \phi+S_{9} \sin ^{2} \theta_{K} \sin ^{2} \theta_{l} \sin 2 \phi\right],
\end{aligned}
$$

where the angular observables $F_{\mathrm{L}}$, the longitudinal polarisation fraction of the $K^{*}, A_{\mathrm{FB}}$, the forwardbackward asymmetry of the dilepton system, and the $S_{i}$ are combinations of the $K^{*}$ amplitudes depending on the WC $C_{7}^{\left({ }^{\prime}\right)}, C_{9}^{\left({ }^{\prime}\right)}, C_{10}^{\left({ }^{\prime}\right)}$ and specific form factors (FF). In Ref. [2] an alternative parametrisation using the $P_{i}^{(\prime)}$ observables was proposed, that are designed such that hadronic FF uncertainties cancel at leading order, with: $P_{2}=2 A_{\mathrm{FB}} / 3\left(1-F_{L}\right) ; P_{4,5,8}^{\prime}=S_{4,5,8} / \sqrt{F_{\mathrm{L}}\left(1-F_{\mathrm{L}}\right)}$; and $P_{6}^{\prime}=S_{7} / \sqrt{F_{\mathrm{L}}\left(1-F_{\mathrm{L}}\right)}$.

\subsection{BABAR analysis of $B \rightarrow K^{*} \ell^{+} \ell^{-}$decays}

Using the full BABAR data sample, $B \rightarrow K^{*} \ell^{+} \ell^{-}$events are reconstructed in five exclusive final states: $B^{+} \rightarrow K^{*+}\left(\rightarrow K_{S}^{0} \pi^{+}\right) \mu^{+} \mu^{-} ; B^{0} \rightarrow K^{* 0}\left(\rightarrow K^{+} \pi^{-}\right) \mu^{+} \mu^{-} ; B^{+} \rightarrow K^{*+}\left(\rightarrow K^{+} \pi^{0}\right) e^{+} e^{-}$; $B^{+} \rightarrow K^{*+}\left(\rightarrow K_{S}^{0} \pi^{+}\right) e^{+} e^{-}$; and $B^{0} \rightarrow K^{* 0}\left(\rightarrow K^{+} \pi^{-}\right) e^{+} e^{-}$. The selection is based on the kinematic variables $m_{\mathrm{ES}} \equiv \sqrt{E_{\mathrm{CM}}^{2} / 4-p_{B}^{* 2}}$ and $\Delta E \equiv E_{B}^{*}-E_{\mathrm{CM}} / 2$, where $p_{B}^{*}$ and $E_{B}^{*}$ are the $B$ momentum 
and energy in the $\Upsilon(4 S)$ center-of-mass $(\mathrm{CM})$ frame, and $E_{\mathrm{CM}}$ is the total CM energy. Random combinations of leptons from semileptonic $B$ and $D$ decays, occurring in both $B \bar{B}$ and $e^{+} e^{-} \rightarrow q \bar{q}$, where $q=u, d, s, c$, events, are reduced using bagged decision trees (BDTs) [3], depending on the considered final state, the background type and the $q^{2}$ region. For both $e^{+} e^{-}$and $\mu^{+} \mu^{-}$modes, the $J / \psi\left(2.85<m_{\ell^{+} \ell^{-}}<3.18 \mathrm{GeV} / c^{2}\right)$ and $\psi(2 S)\left(3.59<m_{\ell^{+} \ell^{-}}<3.77 \mathrm{GeV} / c^{2}\right)$ mass regions are vetoed and used as high-statistics control samples.

The angular variables are extracted in several $q^{2}$ bins [4] from the data using a series of likelihood (LH) fits which proceed in several steps. An initial unbinned maximum LH fit of $m_{\mathrm{ES}}\left(>5.2 \mathrm{GeV} / c^{2}\right), m_{K \pi}$ and a likelihood ratio $L_{R}$ that discriminates against random combinatorial $B \bar{B}$ backgrounds is performed. This step allows to fix all the corresponding probability density function shapes. Then, $F_{L}$ is obtained from a 4-d fit to $m_{\mathrm{ES}}\left(>5.27 \mathrm{GeV} / c^{2}\right), m_{K \pi}, L_{R}$ and $\cos \theta_{K}$ using: $\mathrm{d} \Gamma /\left(\Gamma\left(q^{2}\right) \mathrm{d}\left(\cos \theta_{K}\right)\right)=(3 / 2) F_{\mathrm{L}}\left(q^{2}\right) \cos ^{2} \theta_{K}+(3 / 4)\left(1-F_{\mathrm{L}}\left(q^{2}\right)\right)\left(1-\cos ^{2} \theta_{K}\right)$. Using the fitted value of $F_{L}$ from the previous fit step as input, $A_{\mathrm{FB}}$ is obtained from a similar 4-d fit, in which $\cos \theta_{\ell}$ replaces $\cos \theta_{K}$ as the fourth dimension in the LH function, with: $\mathrm{d} \Gamma /\left(\Gamma\left(q^{2}\right) \mathrm{d}\left(\cos \theta_{\ell}\right)\right)=(3 / 4) F_{\mathrm{L}}\left(q^{2}\right)\left(1-\cos ^{2} \theta_{l}\right)+(3 / 8)\left(1-F_{\mathrm{L}}\left(q^{2}\right)\right)\left(1+\cos ^{2} \theta_{l}\right)+A_{\mathrm{FB}}\left(q^{2}\right) \cos \theta_{l}$.

The results obtained for $F_{L}$ and $A_{\mathrm{FB}}$ in the $q^{2}$ range $1.0<q^{2}<6.0 \mathrm{GeV}^{2} / c^{4}$ are given in Fig. 1. This $q^{2}$ range corresponds to the perturbative window away from the $q^{2} \rightarrow 0$ photon pole and the $c \bar{c}$ resonances at higher $q^{2}$, where theory uncertainties are considered to be under good control. The $B^{0} \rightarrow K^{* 0} \ell^{+} \ell^{-}$results are in reasonable agreement with both SM theory expectations and other experimental results. However, we observe relatively very small values for $F_{\mathrm{L}}$ in $B^{+} \rightarrow K^{*+} \ell^{+} \ell^{-}$, exhibiting tension with both the $B^{0} \rightarrow K^{* 0} \ell^{+} \ell^{-}$results as well as the SM expectations. Using the measured values of $F_{L}$ and $A_{\mathrm{FB}}$, the observable $P_{2}$ is computed in each $q^{2}$ bins, whose results, given in Ref. [4], exhibit an overall good agreement with the SM expectations. The full results of the analysis are published in Ref. [4].

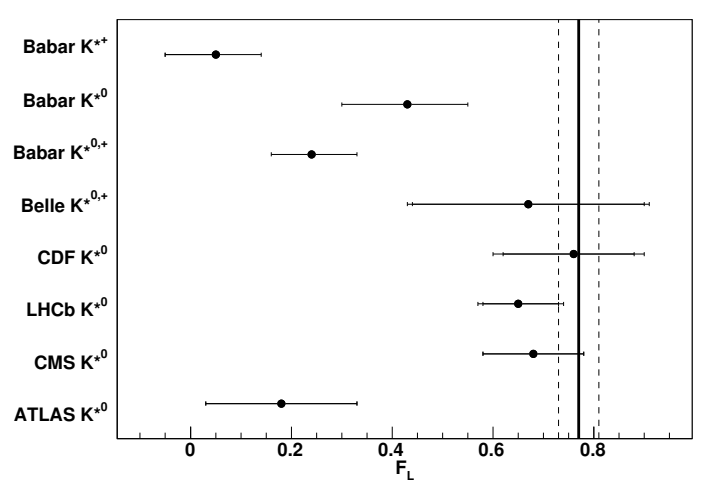

(a) $F_{\mathrm{L}}$.

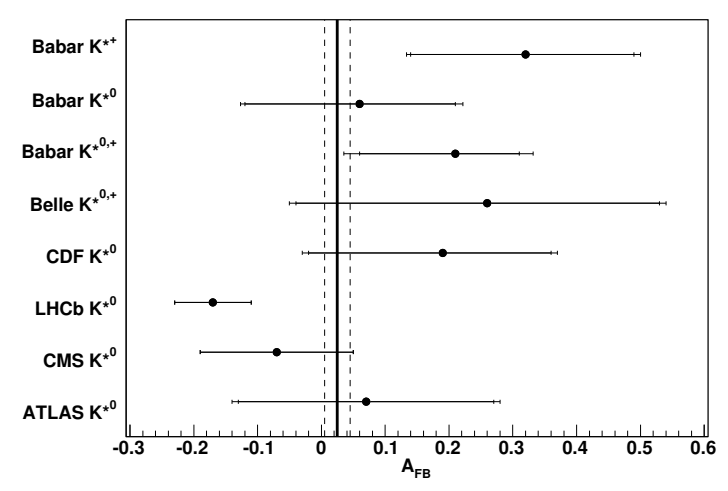

(b) $A_{\mathrm{FB}}$.

Figure 1: $F_{\mathrm{L}}$ (left) and $A_{\mathrm{FB}}$ (right) results in the $q^{2}$ range $1.0<q^{2}<6.0 \mathrm{GeV}^{2} / c^{4}$, along with those of other experiments [5, 6, 17, 8, 9] and the SM expectation (vertical lines) [1, 10, 11, 12, 13, 14].

\subsection{Belle analysis of $B^{0} \rightarrow K^{* 0} \ell^{+} \ell^{-}$decays}

Using the full Belle data sample, $B^{0} \rightarrow K^{* 0} \ell^{+} \ell^{-}$events are reconstructed in two exclusive final states: $B^{0} \rightarrow K^{* 0}\left(\rightarrow K^{+} \pi^{-}\right) \mu^{+} \mu^{-}$and $B^{0} \rightarrow K^{* 0}\left(\rightarrow K^{+} \pi^{-}\right) e^{+} e^{-}$. The present analysis is 
an update of the one published in Ref. [5]. The selection is based on the kinematic variables $M_{\mathrm{bc}} \equiv \sqrt{E_{\mathrm{CM}}^{2} / c^{4}-\left|\vec{p}_{B}^{*}\right|^{2} / c^{2}}$ and $\Delta E$. Doubly misidentified events from $B \rightarrow K^{*} \pi \pi$ when both pions are misidentified as muons, as well as random combinations of leptons from semileptonic $B$ and $D$ decays, occurring in both $B \bar{B}$ and $e^{+} e^{-} \rightarrow q \bar{q}$ events, are reduced using neural network (NN) classifiers [15]. The later use input information from kinematic variables, and from the particle identification system, as well as from event shape variables. The selection requirement for the neural networks are optimized for the sensitivity of the angular analysis using pseudo experiments with simulated data. Similarly to the BABAR analysis (Sec. 2.1) $B \rightarrow K^{*} J / \psi$ and $B \rightarrow K^{*} \psi(2 S)$ events are vetoed and used as high-statistics control samples.

The angular variables are extracted in several $q^{2}$ bins [16] from the data using a series of likelihood (LH) fits which proceed in several steps. First the signal and background are identified from a fit to $M_{\mathrm{bc}}\left(>5.22 \mathrm{GeV} / c^{2}\right)$. The $M_{\mathrm{bc}}$ variable is split into a signal (upper) and sideband (lower) region at $5.27 \mathrm{GeV} / c^{2}$. In the second step the shape of the background for the angular observables is estimated on the $M_{\mathrm{bc}}$ sideband. In the following a folding technique is applied [16, 17], which reduces the number of fitting parameters and hence improves the convergence of the fit. All observables $P_{4,5,6,8}^{\prime}$ are extracted from the data in the signal region using three-dimensional unbinned maximum likelihood fits in each $q^{2}$ bin using the folded signal PDF, fixed background shapes and a fixed number of signal events.

As shown in Fig. 2, for $P_{5}^{\prime}$ a deviation with respect to the DHMV SM prediction [2] is observed with a significance of $2.1 \sigma$ in the $q^{2}$ range $4.0<q^{2}<8.0 \mathrm{GeV}^{2} / c^{4}$. The discrepancy in $P_{5}^{\prime}$ supports measurements by $\mathrm{LHCb}[17,18]$, where a $3.4 \sigma$ deviation is observed in the same $q^{2}$ region. The full results of the analysis are published in Ref. [16].

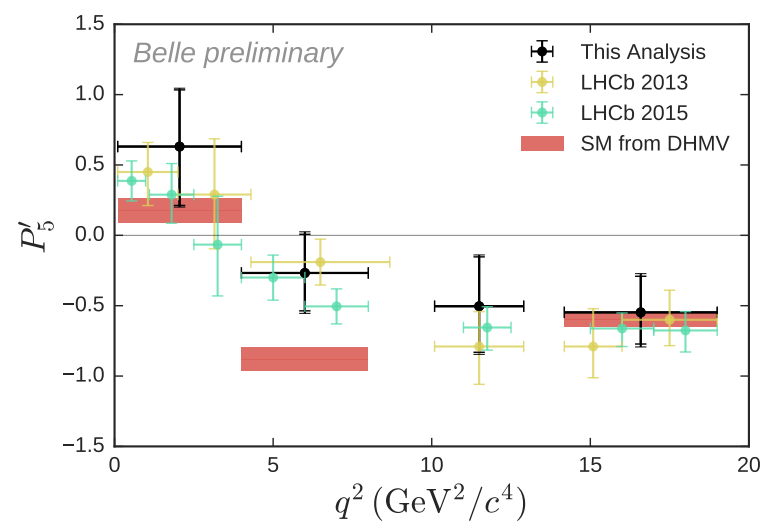

Figure 2: Result for the $P_{5}^{\prime}$ observable compared to Descotes-Genon, Hofer, Matias and Virto (DHMV) SM predictions [2]. Results from $\mathrm{LHCb}[17,18]$ are shown for comparison.

3. Searches for rare $B^{0} \rightarrow \phi \gamma$ and $B^{+} \rightarrow K^{+} \tau^{+} \tau^{-}$decays

\subsection{The decay $B^{0} \rightarrow \phi \gamma$}

In the SM, the decay $B^{0} \rightarrow \phi \gamma$ proceeds through electroweak and gluonic $b \rightarrow d$ penguin annihilation processes, which are highly suppressed with predicted branching fraction in the range 

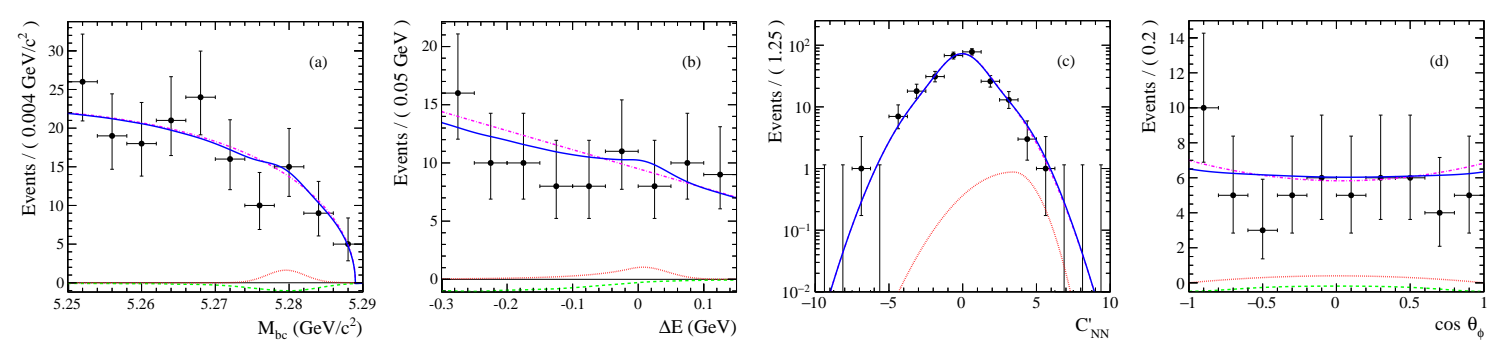

Figure 3: Projections of the four-dimensional fit: (a) $M_{\mathrm{bc}}$; (b) $\Delta E$; (c) $C_{\mathrm{NN}}^{\prime}$; and (d) $\cos \theta_{\phi}$. The points with error bars show the data, the dotted (red) curves represent the signal, the dashed-dotted (magenta) curves represent continuum events, the dashed (green) curves represent the charmless background, and the solid (blue) curves represent the total fit.

$10^{-12}$ to $10^{-11}[19,20]$. The presence of new particles in the internal loop, such as charged Higgs boson or supersymmetric squarks, could enhance the branching fraction to the level of $10^{-9}$ to $10^{-8}$ [19]. Experimentally, no evidence for this decay has been found, and the current upper limit on the branching fraction is $8.5 \times 10^{-7}$ at $90 \%$ confidence level (C.L.) [21].

Using the full Belle data sample, $B^{0} \rightarrow \phi \gamma$ events are reconstructed from the combination of a high-energy photon and a $\phi \rightarrow K^{+} K^{-}$. The photon momentum is required to be in the range $[2.0,2.8] \mathrm{GeV} / c$ in the $\Upsilon(4 \mathrm{~S}) \mathrm{CM}$ frame, while $m_{K^{+} K^{-}}$is required to be in the range $[1.000,1.039] \mathrm{GeV} / c^{2}$ and the two charged tracks to from a common vertex. Background from $\pi^{0}(\eta) \rightarrow \gamma \gamma$ decays are rejected based on likelihood ratios. $B$-meson candidates are selected by requiring $M_{\mathrm{bc}} \in[5.25,5.29] \mathrm{GeV} / c^{2}$ and $\Delta E \in[-0.30,0.15] \mathrm{GeV}$. A NN classifier [15], based on topological variables, is finally added to the selection requirements to help suppressing charmless and continuum events.

The branching fraction of $B^{0} \rightarrow \phi \gamma$ decays is obtained from an unbinned extended maximum likelihood fit to the variables $M_{\mathrm{bc}}, \Delta E$, the $\mathrm{NN}$ output $\left(C_{N N}^{\prime}\right)$, and $\cos \theta_{\phi}$, where $\theta_{\phi}$ is the angle between the $K^{+}$momentum and the opposite of the $B$ flight direction in the $\phi$ rest frame, which provides additional discrimination between signal and continuum events. The projections of the four-dimension fit are shown in Fig. 3. No evidence for this decay is found and an upper limit on the branching fraction of $\mathscr{B}\left(B^{0} \rightarrow \phi \gamma\right)<1.0 \times 10^{-7}$ is set at $90 \%$ C.L. This limit is almost an order of magnitude lower than the previous most stringent result [21]. The full results of the analysis are published in Ref. [22].

\subsection{The decay $B^{+} \rightarrow K^{+} \tau^{+} \tau^{-}$}

The decay $B^{+} \rightarrow K^{+} \tau^{+} \tau^{-}$is the third family equivalent of $B^{+} \rightarrow K^{+} \ell^{+} \ell^{-}$and hence may provide additional sensitivity to new physics due to third-generation couplings and the large mass of the $\tau$ lepton [23]. The analysis uses hadronic $B$ meson tagging techniques, where one of the two $B$ mesons, referred to as the $B_{\mathrm{tag}}$, is reconstructed exclusively via its decay into one of several hadronic decay modes. The remaining tracks, clusters, and missing energy in the event are attributed to the signal $B$, denoted as $B_{\text {sig }}$. Only leptonic decays of the $\tau$ are considered: $\tau^{+} \rightarrow e^{+} v_{e} \bar{v}_{\tau}$ and $\tau^{+} \rightarrow \mu^{+} v_{\mu} \bar{v}_{\tau}$, which results in three signal decay topologies with a charged $K$, multiple missing neutrinos, and either $e^{+} e^{-}, \mu^{+} \mu^{-}$or $e^{+} \mu^{-}$in the final state. Since the $B_{\mathrm{tag}}$ is fully reconstructed, 


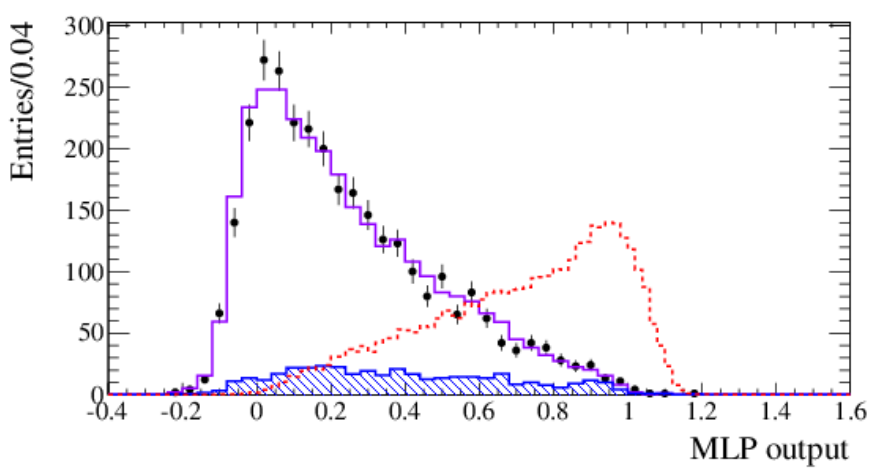

Figure 4: (color online) MLP output distribution for the three signal channels combined. The $B^{+} \rightarrow$ $K^{+} \tau^{+} \tau^{-}$signal MC distribution is shown (dashed) with arbitrary normalization. The data (points) are overlaid on the expected combinatorial (shaded) plus $m_{\mathrm{ES}}$-peaking (solid) background contributions.

its four-vector is fully determined and thus that of the $B_{\text {sig }}$ can be calculated. $B_{\text {tag }}$ candidates are selected requiring $5.20<m_{\mathrm{ES}}<5.30 \mathrm{GeV} / c^{2}$ and $-0.12<\Delta E<0.12 \mathrm{GeV}$, and $B^{+} \rightarrow K^{+} \tau^{+} \tau^{-}$ signal events are required to have a charged $B_{\text {tag }}$ candidate with $m_{\mathrm{ES}}>5.27 \mathrm{GeV} / c^{2}$ and a non-zero missing energy. $B^{+} \rightarrow K^{+} \tau^{+} \tau^{-}$candidates are required to possess exactly three charged tracks satisfying particle identification requirements consistent with one charged $K$ and an $e^{+} e^{-}, \mu^{+} \mu^{-}$, or $e^{+} \mu^{-}$pair, and the $K^{ \pm}$is required to have a charge opposite to that of $B_{\mathrm{tag}}$. Continuum events are suppressed using a multivariate likelihood selector based on event-shape variables, and vetoes are applied to remove backgrounds with a $J / \psi\left(3.00<m_{\ell^{+} \ell^{-}}<3.19 \mathrm{GeV} / c^{2}\right)$ or a $D^{0}\left(1.80<m_{K^{-} \ell^{+}}<\right.$ $\left.1.90 \mathrm{GeV} / c^{2}\right)$.

The main remaining background, with a final state identical to that of signal, originates from $B \bar{B}$ events in which a properly reconstructed $B_{\text {tag }}$ is accompanied by $B_{\text {sig }} \rightarrow D^{(*)} \ell \bar{v}_{\ell}$, with $D^{(*)} \rightarrow$ $K \ell^{\prime} \overline{\boldsymbol{v}}_{\ell^{\prime}}$. These contributions are suppressed using a multi-layer perceptron (MLP) neural network [24] based on kinematic and event shape variables. Figure 4 shows the MLP distribution, which output is required to be $>0.70$ for the $e^{+} e^{-}$and $\mu^{+} \mu^{-}$channels and $>0.75$ for the $e^{+} \mu^{-}$ channel.

The branching fraction for each of the signal modes is defined as $\mathscr{B}_{i}=\left(N_{\mathrm{obs}}^{i}-N_{\mathrm{bkg}}^{i}\right) /\left(\varepsilon_{\mathrm{sig}}^{i} N_{B \bar{B}}\right)$, where $N_{B \bar{B}}$ is the total number of $B \bar{B}$ pairs, $N_{\mathrm{obs}}^{i}$ is the number of observed events after the selection, $\varepsilon_{\text {sig }}^{i}$ the signal efficiency, and $N_{\mathrm{bkg}}^{i}$ the background estimate, obtained from simulation. The final signal efficiencies, background estimates and observed yields of each signal mode are given in

\begin{tabular}{lccc}
\hline \hline & $e^{+} e^{-}$ & $\mu^{+} \mu^{-}$ & $e^{+} \mu^{-}$ \\
\hline \hline$N_{\text {bkg }}^{i}$ & $49.4 \pm 2.4 \pm 2.9$ & $45.8 \pm 2.4 \pm 3.2$ & $59.2 \pm 2.8 \pm 3.5$ \\
$\varepsilon_{\text {sig }}^{i}\left(\times 10^{-5}\right)$ & $1.1 \pm 0.2 \pm 0.1$ & $1.3 \pm 0.2 \pm 0.1$ & $2.1 \pm 0.2 \pm 0.2$ \\
$N_{\text {obs }}^{i}$ & 45 & 39 & 92 \\
Significance $(\sigma)$ & -0.6 & -0.9 & 3.7 \\
\hline \hline
\end{tabular}

Table 1: Expected background yields, $N_{\mathrm{bkg}}^{i}$, signal efficiencies, $\varepsilon_{\mathrm{sig}}^{i}$, number of observed data events, $N_{\mathrm{obs}}^{i}$, and signed significance for each signal mode. Quoted uncertainties are statistical and systematic. 
Table 1, with the associated branching fraction significance. No significant signal is observed and the upper limit on the final branching fraction for the combined three modes is determined to be $2.25 \times 10^{-3}$ at the $90 \%$ C.L. The full results of the analysis are published in Ref. [25].

\section{Conclusion}

The BABAR and Belle Collaborations, several years after the shutdown of the experiments, are still producing competitive results, especially for channels involving neutral particles such as $\pi^{0}$ or $K_{S}^{0}$, and $\tau$ leptons. Four recent analyses have been presented, for which all the results are in agreement with the SM predictions.

\section{References}

[1] W. Altmannshofer et al., JHEP 0901, 019 (2009)

[2] S. Descotes-Genon, L. Hofer, J. Matias and J. Virto, JHEP 1412, 125 (2014)

[3] L. Breiman, Mach. Learn. 24, 123 (1996); I. Narsky, physics/0507157 (2005)

[4] J. P. Lees et al. [BaBar Collaboration], Phys. Rev. D 93, 052015 (2016)

[5] J.-T. Wei et al. [Belle Collaboration], Phys. Rev. Lett. 103, 171801 (2009)

[6] T. Aaltonen et al. [CDF Collaboration], Phys. Rev. Lett. 108, 081807 (2012)

[7] R. Aaij et al. [LHCb Collaboration], JHEP 1308, 131 (2013)

[8] S. Chatrchyan et al. [CMS Collaboration], Phys. Lett. B 727, 77 (2013)

[9] G. Aad et al. [ATLAS Collaboration], ATLAS-CONF-2013-038

[10] G. Buchalla, A. J. Buras and M. E. Lautenbacher, Rev. Mod. Phys. 68, 1125 (1996)

[11] F. Kruger, L. M. Sehgal, N. Sinha and R. Sinha, Phys. Rev. D 61, 114028 (2000) [Erratum-ibid. D 63, 019901 (2001)]

[12] A. Ali, E. Lunghi, C. Greub and G. Hiller, Phys. Rev. D 66, 034002 (2002)

[13] K. S. M. Lee, Z. Ligeti, I. W. Stewart and F. J. Tackmann, Phys. Rev. D 75, 034016 (2007)

[14] A. Hovhannisyan, W. S. Hou and N. Mahajan, Phys. Rev. D 77, 014016 (2008)

[15] M. Feindt and U. Kerzel, Nucl. Instrum. Meth. A 559, 190 (2006)

[16] A. Abdesselam et al. [Belle Collaboration], arXiv:1604.04042 [hep-ex]

[17] R. Aaij et al. [LHCb Collaboration], Phys. Rev. Lett. 111, 191801 (2013)

[18] R. Aaij et al. [LHCb Collaboration], JHEP 1602, 104 (2016)

[19] J. Hua, C. S. Kim and Y. Li, Eur. Phys. J. C 69, 139 (2010)

[20] C. D. Lu, Y. L. Shen and W. Wang, Chin. Phys. Lett. 23, 2684 (2006)

[21] B. Aubert et al. [BaBar Collaboration], Phys. Rev. D 72, 091103 (2005)

[22] Z. King et al. [Belle Collaboration], Phys. Rev. D 93, 111101 (2016)

[23] L. Calibbi, A. Crivellin and T. Ota, Phys. Rev. Lett. 115, 181801 (2015)

[24] B. H. Denby, Neural Comput. 5, 505 (1993)

[25] J. P. Lees et al. [BaBar Collaboration], arXiv:1605.09637 [hep-ex] 